\title{
Cut and break verbs in Yélî Dnye, the Papuan language of Rossel Island*
}

STEPHEN C. LEVINSON

\section{Abstract}

The paper explores verbs of cutting and breaking (C\&B, hereafter) in Yélî Dnye, the Papuan language of Rossel Island. The Yélî Dnye verbs covering the $C \& B$ domain do not divide it in the expected way, with verbs focusing on special instruments and manners of action on the one hand, and verbs focusing on the resultant state on the other. Instead, just three transitive verbs and their intransitive counterparts cover most of the domain, and they are all based on 'exotic' distinctions in mode of severance-coherent severance with the grain vs. against the grain, and incoherent severance (regardless of grain).

Keywords: cut and break; separation events; verb semantics; Yélî Dnye; Papuan languages; language and culture.

\section{Background}

It is intriguing to wonder what the cutting terminology of a stone age people would have looked like. Perhaps some insight can be gained by looking at the vocabulary used in this domain by a language group that has only had metal instruments in recent historical time. ${ }^{1}$

Yélî Dnye is an isolated so-called Papuan language, not known to be related to any other language, spoken by 4000 odd souls on the easternmost island of the Louiseade Archipelago in a remote location. The language is phonetically, morphologically, and syntactically highly complex and irregular. It is morphologically and syntactically ergative. There are large sets of inflectional affixes or clitics, and verbs supplete on many varying dimensions. The language is described in Henderson (1995), Henderson and Henderson (1999), and Levinson (in prep.).

Cutting and breaking (C\&B, hereafter) terms have a cultural ecology of course, and it is relevant to know that Rossel Islanders make houses and 
outrigger sailing canoes entirely out of the wood, vines and leaves of the forest. They cultivate taros, yams, plantains and sago as traditional crops, supplemented with fish and pork. ${ }^{2}$

In the crosslinguistic sample represented in this special issue, Yélî Dnye stands out as unusual in certain respects. A reason for this is that although the relevant vocabulary is quite restricted, the core semantic distinctions made by Yélî Dnye in this domain are quite different. In a nutshell, there is one main general breaking verbal notion, and two core verbal notions that fall within a cutting domain-one indicates severing across the grain, and the other splitting/cutting/tearing along the grain. These are verbal notions rather than verbs, since they are (with one exception) each encoded in two different verbs: a transitive, and an underived intransitive root. Furthermore each of these roots is in fact a collection of suppletive forms. Thus, the corresponding lexicography and syntax turns out not to be simple at all. In addition to these basic notions, there are a number of more specialized forms meaning such things as 'cutting open', 'cutting repeatedly, chopping', 'felling trees', 'slashing', and so forth, but these are of nothing like the same frequency or salience.

\section{The core verbs of $C \& B$}

The semantic domain of focal interest can be thought of as "caused division", where an agent causes an object (the theme) to lose its integrity (wholeness), with or without the use of a tool or instrument. A supposition is that most languages will have a set of basic verbs that together exhaustively cover this domain at a general level, supplemented with more detailed verbs which describe subtypes of these actions (cf. general break vs. specific crush; general cut vs. specific cleave).

The core verbs are here operationally defined as those occurring in responses to the "Cut and Break Clips" (a set of video stimuli that participants described $)^{3}$, but there is no doubt that they are by far the most frequent verbs covering (most of) this domain. Since Yélî Dnye verbs are suppletive, we give here all the parts (in these cases suppleting over tense, aspect and mood). ${ }^{4}$ On various grounds, the transitive and intransitive counterparts are not here treated as suppletive parts of the same verb, but as distinct verbs in their own right. Thus, most Yélî Dnye verbs come in doublets of transitive and intransitive form (on alternations see Section 2.2), but here one of the focal verbs does not. There are thus 5 focal verbs or 2.5 doublets which are presented in the following tables (glosses approximate): 
Table 1. Transitive verb with intransitive counterpart: 'break's

\begin{tabular}{|c|c|c|c|}
\hline \multicolumn{2}{|c|}{ 'break something' (transitive) } & \multicolumn{2}{|c|}{ 'break' (intransitive) } \\
\hline Tense/Aspect/Mood & Root & Tense/Aspect/Mood & Root \\
\hline TV citation form & pwââ & IV citation form & рwópu \\
\hline PUNCT.IMPERATIVE & pwaa ngi & PUNCT.IMPERATIVE & pwédi! \\
\hline PUNCT.PROX.PAST & $p w a \hat{a} / p u w \hat{a}$ & PUNCT.PROX.PAST & pwópu \\
\hline PUNCT.REM.PAST & pwââapuwâa & PUNCT.REM.PAST & pwaa wo \\
\hline FOLLOWED & pwaa wo & FOLLOWED & pwaa wo \\
\hline CONTINUOUS & pwaapî & CONTINUOUS & pwópupwópu \\
\hline
\end{tabular}

Table 2. Transitive verb with intransitive counterpart: 'sever along the grain: split, tear'6

\begin{tabular}{|c|c|c|c|}
\hline \multicolumn{2}{|c|}{ 'split something' (transitive) } & \multicolumn{2}{|c|}{ 'split' (intransitive) } \\
\hline Tense/Aspect/Mood & Root & Tense/Aspect/Mood & Root \\
\hline TV citation form & chaa & IV citation form & chópu \\
\hline PUNCT.IMPERATIVE & chaa ngi & PUNCT.IMPERATIVE & chépi! \\
\hline PUNCT.PROX.PAST & chaa & PUNCT.PROX.PAST & chapîlchaa \\
\hline PUNCT.REM.PAST & chópu & PUNCT.REM.PAST & chópu \\
\hline FOLLOWED & (n.a.) & FOLLOWED & (n.a.) \\
\hline CONTINUOUS & chapî & CONTINUOUS & chópuchópu \\
\hline
\end{tabular}

Table 3. Transitive verb: 'sever across the grain: cut, chop, sunder'

\begin{tabular}{ll}
\hline \multicolumn{2}{c}{ 'cut something' (transitive) } \\
\hline Tense/Aspect/Mood & Root \\
\hline TV citation form & châpwo \\
PUNCT.IMPERATIVE & chepwe \\
PUNCT.PROX.PAST & châpwo \\
PUNCT.REM.PAST & châpwo \\
FOLLOWED & (n.a.) \\
CONTINUOUS & châpwo \\
\hline
\end{tabular}

These verbs are used to describe the great majority of the C\&B scenes. In Section 3 we will discuss other verbs in the same domain.

\subsection{The semantics of the core verbs}

Some of the Yélî Dnye verbs are unusual viewed against the general tendencies emerging from comparative work (see Majid et al., this issue). First, no distinction is made between snapping and smashing events, thus explaining the low correlation on Dimension 3. Second, the verb 
chaa only partially correlates with Dimension 2 . This is a clue, if it was needed, that these verbs represent an unusual categorization of this domain. For strictly speaking there is no "cutting" verb in the language! The way the language semantically divides the domain seems to be as follows, with the numbered scenarios for each of the three transitive verbs subsumed within the indicated general notion (CAPS), and thus not recognized as different senses:

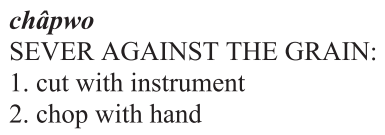

\section{chaa}

SEVER WITH THE GRAIN:

1. split with instrument

2. tear by hand without instrument

\section{pwââ}

BREAK (divide incoherently, regardless of grain)

1. with sharp instrument

2. with blunt instrument

3. with hands

Figure 1. Intensions of the main verbs of $C \& B$

The crucial underlying semantic parameter appears to be the notion of grain, more exactly fibers. Materials which are built of aligned fibers (wood, leafs, vines, cloth, etc.) have the property that they are severable in very different ways, that is 'with the grain' (along the fibers), or 'against the grain' (across the fibers). These materials in turn differ from those without 'grain', i.e., not built from fibers, which can easily break incoherently in any direction (fibrous materials can also break incoherently under extreme compression or torsion). These underlying distinctions in folk "materials science" seem to underlie the distinction between our three transitive verbs: on the one hand, wood can undergo chaa 'splitting along the grain', or châpwo 'severing across the grain', or pwâa 'cracking both along and across the grain'; on the other hand, cloth will tend to chaa 'tear or split', and pottery to pwâa 'break into irregular pieces'.

All three distinctions are concerned primarily with the state change caused in the theme (the affected object), not with the type of activity that produces it. In a sense, they are semantically all break-like verbs, caring primarily for how the theme breaks: along the grain, across the grain or less systematically. Alternatively, one could think about them as three "divide" verbs: divide coherently into two along the grain vs. divide coherently across the gain vs. divide incoherently. This three-way distinction recognizes no special role for an instrument of any kind, let alone making 

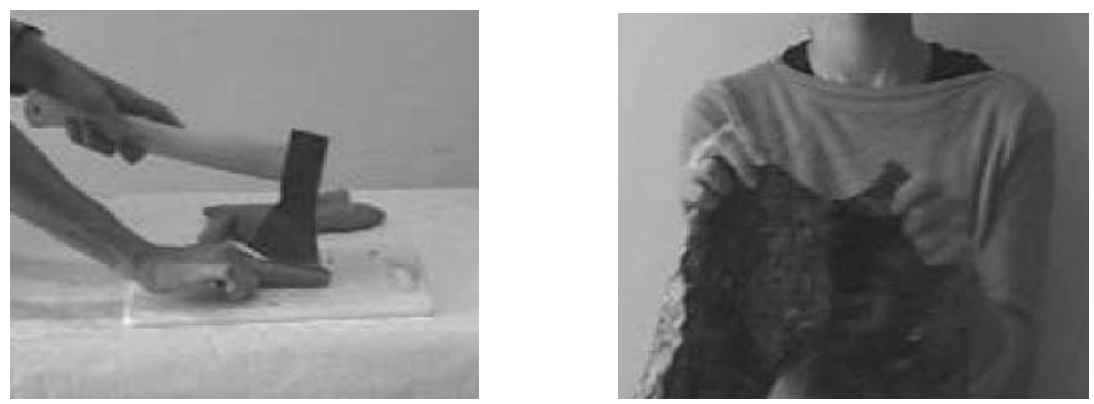

chaa 'sever with grain'

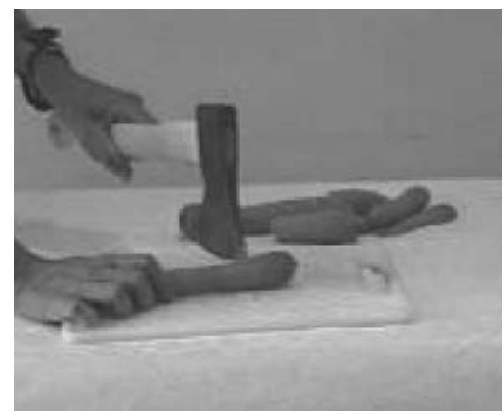

châpwo 'sever against grain'

Figure 2. Some clips illustrating the 'sever with grain' vs. 'sever against the grain' distinction

distinctions between say axes, saws, and knives. Note especially how in Figure 1, the verb chaa applies to both tearing scenes and scenes depicting, e.g., lengthwise division with a knife.

The C\&B stimulus set includes some nice minimally contrasting scenes that make these distinctions crystal clear. For example, there are two scenes (see Figure 2) where an axe is used to divide a carrot: lengthwise division is chaa 'sever along the grain', across-wise division is châpwo, 'sever across the grain' (similarly for knife cutting along vs. across a carrot). A scene where an axe is used to cut across a carrot is classified with châpwo, as are all karate-chop scenes, where the edge of the hand is used to effect the separation, making clear that this apparent cutting verb has in fact nothing to do with instruments. The classification as chaa of tearing scenes with lengthwise cutting scenes (knife or axe used to cut a carrot lengthwise) is also clear. Another scene which gets chaa is quite revealing. A mallet strikes a cloth held taut between clamps: the first strike splits it nearly into two, the next completes the division. The cloth is clearly dividing systematically along the grain. In contrast in a different scene the 
same mallet strikes a string held between the same clamps and snaps it. Now the scene gets $p w a \hat{a} \hat{a}$, 'break', 'divide incoherently'.

Further evidence for the importance of these semantic parameters can be found beyond the core basic set of Yélî Dnye verbs. Thus, châpwo means 'sever across the grain', and the causative châpwo kwolo is formed by embedding the gerund as the theme-argument of the causative verb $k w o l o$. This verb has the specialized meaning 'sever crosswise into many pieces'. It contrasts with another causative form pepe kwolo which means 'sever longitudinally into many pieces' (there is no synchronic independent meaning of pepe).

This three-way semantic distinction clearly cuts across any cutting/ breaking division of the domain. It is an entirely different way to divide such events. Why would the inhabitants of Rossel choose another system? Probably because the language reflects the culture of a century ago, when there were no metal tools, and the only substantial tools were blunt stone axes ground from basalt. With such simple tools, the bush materials from which Rossels construct canoes and houses could only be made with difficulty. Cutting across the grain was especially problematic, and wherever possible timber, vines, and fibers were divided along the grain. There is still a material culture of split fibres-floor boards, baskets, thatch, planked canoes, and ropes all involve split materials. The Yélî Dnye semantics serves as a useful reminder that "universal" tendencies in semantics are perhaps just as likely to reflect cultural tendencies as any nativist constraints (just in case anyone would be silly enough to suggest that "cutting" was an innate idea, remember that our species left Africa with only the simplest tool kit).

The English vocabulary of $\mathrm{C} \& \mathrm{~B}$ is greatly expanded through distinctions between instruments used (cut, saw, chop, scythe), the manner employed (hack, hew, slash, gash) or both (cleave, stab, lop). We have already seen that Yélî makes no distinctions according to instrument- to code the instrument an NP in instrumental case is employed, and all three transitive verbs collocate happily with such an NP meaning 'with the knife', 'with the hammer', etc. Interestingly, consultants as often as not felt it unnecessary to encode the instrument. Yélî Dnye is also oblivious, as it were, to manner distinctions. In the C\&B stimuli, a number of scenes would have natural English descriptions of the kind hack, smash or shatter, where the verbs encode manner distinctions, but these pass without comment on manner in Yélî Dnye. Only under prompting could one extract the adverbial dpodo mbiy:e 'with effort', or the construction yeda pwââ ala pwâa 'keep on breaking'. A parallel could here be drawn to the well known satellite-framing vs. verb-framing distinction in motion semantics (Talmy 2000), where only the former (e.g., Germanic lan- 
guages like English) permit merging of manner information with the main verb. ${ }^{7}$

\subsection{Semantics, argument-structure and syntax of the core verbs ${ }^{8}$}

It has long been noted that the C\&B domain seems to lie across a conceptual fault-line, which shows up as soon as the constructions these verbs participate in are investigated further. In particular, break verbs undergo the causative-inchoative alternation (as in He broke the pot vs. The pot broke), while cut verbs do not (He cut the cake vs. ?The cake cut). The reverse distribution goes for the conative alternation, specialized to cut verbs (He cut the cake vs. He cut at the cake, compared to He broke the pot vs. *He broke at the pot). There are said to be analogs to these alternations, and the systematic absence of them, in many languages (Hale and Keysar 1987). The underlying semantic difference is thought to be that cut verbs focus on the activity which brings about division, while break verbs focus on the resulting state.

In view of the above analysis of the semantics of Yélî Dnye core verbs in this domain, we might predict that any alternations undergone by these verbs should (a) be those specialized to break verbs, i.e., the causativeinchoative alternation, and (b) apply to all three of the core transitive verbs, regardless of whether they seem to translate as cut verbs in English.

Here are the facts. Yélî Dnye is ergative, and transitive verbs take a case-marked ergative subject and unmarked absolutive object, while intransitive verbs take an absolutive (unmarked) subject. Since most verbs come in doublets, with a transitive and intransitive root (sometimes quite unrelated in form), valence-changing operations (or alternations) are small in number and limited in use.

All NPs can be omitted if recoverable in context, but the ergative NP is often omitted to give a pseudo-passive reading. Thus, where a man breaks a carrot with a hammer, we have the description of the action in (1a) and the result in (1b) using the pseudo-passive:
a. pi ngê hammer ngê k:aa dê
person ERG hammer INSTR taro 3SIMMPASTPUNCT
pwââ.
broke.trans
'The man broke the taro with a hammer.'
b. k:aa dê pwââ hammer ngê.
taro 3SIMMPASTPUNCT broke hammer INSTR
'The taro got broken with a hammer.'

There are two main other ways of talking about the resultant state, as indicated in (2b) and (2c) below: 

(2) a. pyââ ngê d:âa dê pwâa.
woman ERG clay.pot 3SIMMPASTPUNCT broke.trans 'The woman broke the pot.'
b. d:ââ dê pwópu.
clay.pot 3SIMMPASTPUNCT broke.intrans
'The pot broke.' (intransitive)
c. d:ââ pwaa ngmê
clay.pot break.trans RESULT
'The pot is broken.' (resultative construction)

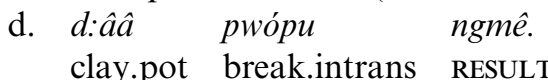
'The pot is broken.' (resultative construction on intransitive root)

Example (2b) uses the intransitive root doublet of pwâa to form an intransitive clause without any kind of syntactic or morphological alternation. However, the (2c) form is a true alternation of the kind often invoked in discussions of this sort: it could indeed be called the inchoative alternate. The resultant form is untensed (unlike (2b)), and preserves only the absolutive argument as subject. With some interesting exceptions, this alternation applies only to transitive punctual verbs, and in fact applies equally to our three key verbs pwââ, chaa, châpwo.

This unified behavior does indeed seem to confirm the prediction, namely that all of the core Yélî verbs have the same underlying semantic structure. They are all verbs of breaking, and thus undergo the same alternations. This is unlike English, which has a separate class of cutting verbs.

Incidentally, one can also take an intransitive verb, causativize it so it is transitive and then apply the resultative alternation. But a few exceptional intransitives, mostly it seems those with some kind of inchoative semantics already (e.g., 'get ripe'; 'become big'), do permit the use of the same resultative construction without prior transitivization. One of them is pwópu, intransitive 'break', as in (2d). Examples (2b) and (2d) would contrast slightly in semantics in that (2b) is tensed and suggests a specific causative action, while (2d) is untensed, nominalized, and emphasizes the state. But now we also see a subtle difference between the core verbs. One of the severing verbs, châpwo, has no intransitive doublet, while the other, chaa has an intransitive doublet chópu which does not take the resultative:

(3) a. yi mbwi chaa ngmê.

tree spine split.trans RESULT

'The stick is split.' (resultative construction) 


\section{b. *yi mbwi chópu ngmê.}

tree spine split.intrans RESULT

'The stick is split.' (resultative construction on intransitive root)

Whether this shows anything about an underlying semantic difference between the three core transitive verbs remains a question for future research.

Pursuing the question of underlying semantic properties, we may ask whether entailment relations show anything about the event representation encoded in these verbs. For example, it has been noted that one can say John cut the lemon but didn't cut through it, but not (or not as easily) John broke the vase, but did not break it in pieces/entirely - a distinction attributable once again to the respective activity vs. result foci. In the case of the Yélî Dnye verbs, there is no such distinction: for example partial tearing of cloth was described as in (4a), while partial breaking of stick (i.e., cracking without separation) was described by (4b):

$$
\begin{array}{lll}
\text { a. pyââ ngê kpîdî dê } & \text { chaa, daa } \\
& \text { woman ERG cloth 3SIMMPASTPUNCT } & \text { split, not } \\
\text { d:ud:u mbiy:e. } & & \\
\text { complete } & \text { ADV'ZER }
\end{array}
$$

'The woman tore the cloth (severed along the grain), but not completely.'

b. pyââ ngê yi mbwii dê

woman ERG tree spine 3SIMMPASTPUNCT

pwââ, daa d:ud:u mbiy:e.

break, not complete ADV'zER

'The woman broke the stick, but not completely.'

The fact that both the 'sever along the grain' verb (describing various cutting scenes) and the 'break' verb show the same pattern reinforces the analysis that they have similar underlying semantics. One could query whether the deniability of the end state in both cases raises doubts about the break-type analysis, that is, a focus on resulting state rather than on an activity. But it seems clear that in neither case is it being asserted that there was an activity of tearing or an activity of breaking which failed to result in the expected end state. Rather, what is asserted is that the end state can be seen to have a number of sub-states or stages, and only some of these have been achieved.

\section{Other verbs in the same domain}

It would be wrong to give the impression that Yélî Dnye is a language with impoverished vocabulary either generally or in this domain. There 
are more specific verbs available. Some of these surfaced in the C\&B task. For example, a scene where a girl pierces a cloth with a stick was described with the transitive verb taa 'to perforate, make a hole by plunging a stick', which itself contrasts with $n g \hat{\imath}$ 'to make a hole by drilling'. Similarly, a scene where a woman cuts a watermelon with a knife but fails to cut through it, was described with the verb nyime, which means something like 'slice' (the example is germane to the prior paragraph):

a. pyââ ngêe mbwaa n:êe da
woman ERG watermelon half 3SIMMPASTPUNCTDEICT
nyime, ngmênê doo chópu
slice, but $\quad$ 3SIMMPASTNEG split.intrans,
doo chaa ngê
3SIMMPASTNEG split.trans 3SSUBJ3SOBJIMMPASTNEG
'The woman sliced the watermelon, but did not split
(intransitive), or split (transitive) it.'

In addition there are specialized verbs for felling trees ( $k p$ :anê, transitive), cutting grass (chiyé, intransitive), slashing cane (ghâa, transitive, 'cut vertical plants in one stroke'), and so forth.

\section{Conclusions}

The Yélî Dnye verbs covering the C\&B domain do not divide it in the expected way, with specialist verbs focusing on instruments and manners of action on one hand, and verbs focusing on the resultant state on the other. Instead, just three transitive verbs and their intransitive counterparts cover most of the domain, and they are all based on 'exotic' distinctions in the mode of severance - namely coherent severance with the grain vs. against the grain, on one hand, and incoherent severance (regardless of grain) on the other. Thus, unlike English, there is no underlying fault line across the domain which divides cutting verbs from breaking verbs. Consequently, the Yélî Dnye verbs appear to behave more or less uniformly from a syntactic point of view. All this accords with a material culture based on fibers and the relatively recent introduction of steel cutting tools. These facts explain why Yélî Dnye appears in one way or another to be unusual in large scale crosslinguistic comparisons (Bohnemeyer, this issue; Majid et al., this issue). 


\section{Notes}

* Max Planck Institute for Psycholinguistics, Postbus 310, Nijmegen, 6525XD, The Netherlands. Author's e-mail address 〈Stephen.Levinson@mpi.nl〉.

1. The first systematic trade in steel tools (axes and knives) started in 1905. Today the metal bush knife and metal axe are essential tools. The island offers no sharp stone like flint or obsidian.

2. Pigs are cut up for distribution at feasts following an exact system of divisions. I have no idea how, or if, this process was carried out before the acquisition of iron tools.

3. Developed by Bohnemeyer et al. (2001). See description in introduction to this special issue (Majid et al., this issue).

4. The $\mathrm{C} \& \mathrm{~B}$ responses analyzed here are from a single subject (following the procedure outlined in Majid et al. this issue). However, the responses are consistent with the pattern of responses from a larger number of speakers (adult and children) to another, related stimulus set, known as "Kids' Cut \& Break" (collected by Penelope Brown), and also with extended textual and elicitation data.

5. Abbreviations: 3s — 3rd singular; ADV'ZER — adverbializer; DEICT — deictic; ERG — ergative; FOLLOWED - special form of the verb co-occurring with inflectional enclitic; IMM - immediate; INSTR - instrument; intrans - intransitive; NEG - negation; OBJ-object; PROXproximate; PUNCT — punctual; REM — remote; SUBJ—subject; trans-transitive.

6. Any resemblance of this verb to English chop is entirely accidental.

7. But, again under prompting, a bi-clausal construction could be used to get around the constraint. Thus, a scene where a man smashes a carrot with a hammer, was described first as 'the man breaks ( $p w a \hat{a} \hat{)}$ ) the taro with a hammer', and under prompting with the additional clause 'the man struck (mgéé) the carrot with a hammer'. The verb mgéé does seem to encode 'hit hard' but does not encode the C\&B result.

8. This section is indebted to clarification of the issues in Bohnemeyer (this issue).

\section{References}

Bohnemeyer, Jürgen

this issue Morpholexical relatedness and the argument structure of verbs of cutting and breaking. Cognitive Linguistics 18(2), 153-177.

Bohnemeyer, Jürgen, Melissa Bowerman, and Penelope Brown

2001 Cut and break clips. In Levinson, Stephen C., and N. J. Enfield (eds.), Field Manual 2001, Language and Cognition Group, Max Planck Institute for Psycholinguistics. Nijmegen: MPI, 90-96.

Hale, Kenneth L., and Samuel J. Keyser

1987 A View from the Middle. (Lexicon Project Working Papers 10.) Cambridge, MA: Center for Cognitive Science, MIT Press.

Henderson, Jim

1995 Phonology and grammar of Yele, Papua New Guinea. Pacific Linguistics, B-112.

Henderson, Jim, and Anne Henderson

1999 Nt:u kópu dyuu u puku dmi (Rossel-English Dictionary). Ukarumpa: SIL Printroom.

Levinson, Stephen C.

in prep. A Grammar of Yélî Dnye. Unpublished MS. 
218 S. C. Levinson

Majid, Asifa, Melissa Bowerman, Miriam van Staden, and James S. Boster this issue The semantic categories of cutting and breaking events: A crosslinguistic perspective. Cognitive Linguistics 18(2), 133-152.

Talmy, Leonard

$2000 \quad$ Toward a Cognitive Semantics. Cambridge, MA: MIT Press. 